EPJ Web of Conferences 32, 01004 (2012)

DOI: $10.1051 /$ epjconf/20123201004

(C) Owned by the authors, published by EDP Sciences, 2012

\title{
ECRH and ECCD scenarios for W7-X
}

\author{
N. B. Marushchenko a , C. D. Beidler, V. Erckmann, J. Geiger, P. Helander, H. P. Laqua, H. Maassberg, \\ and Y. Turkin
}

Max-Planck-Institut für Plasmaphysik, IPP-EURATOM Association, D-17491 Greifswald, Germany

\begin{abstract}
The main ECRH scenarios for the W7-X Stellarator are described. Both X2 (low and moderate densities) and $\mathrm{O} 2$ scenarios (high density) have been studied. Since O2 scenario cannot be realized without pre-heating, transition from X2 to $\mathrm{O} 2$ scenarios has been discussed. Due to a lack of Ohmic transformer, only ECCD is available for compensating the bootstrap current and for controlling the edge rotational transform value. The efficiency of ECCD for all main scenarios has been estimated. All simulations have been performed by a 1D transport code coupled self-consistently with ray-tracing code.
\end{abstract}

\section{Introduction}

The W7-X stellarator [1] is a large-scale device $\left(R_{0} \simeq 5.5 \mathrm{~m}\right.$, and $\left.a \simeq 0.53 \mathrm{~m}\right)$ with superconducting coils and without an ohmic transformer. The main scientific objective is to demonstrate good plasma confinement in steady-state operation with reactor-relevant parameters. The magnetic equilibrium (a low-shear configuration of Helias type with five field periods) is quite flexible and well optimized with respect to low neoclassical transport (the "standard" configuration, with the variation of magnetic field along the axis $B_{\max } / B_{\min }=1.09$ and edge value $t_{a} \simeq 5 / 4$ ) and small bootstrap current (the "highmirror" configuration, with $B_{\max } / B_{\min }=1.22$ and $t_{a} \simeq 1$ ) [2].

The system for electron cyclotron resonance heating (ECRH) is designed for continuous operation with a total injected power up to $10 \mathrm{MW}$ at $140 \mathrm{GHz}$ [3]. The main ports for RF-power launch are situated near the "bean-shaped" plane where the maximum of $B$ is located which guarantees a minimization of heating of the trapped electrons.

For low and moderate densities up to $n_{e}<1.2 \times 10^{20} \mathrm{~m}^{-3}$, the main heating scheme is low-field-side launch of the RF beams at the 2nd harmonic of the extraordinary mode (X2 scenario). High-density regimes above the X2 cut-off density, from $1.2 \times 10^{20} \mathrm{~m}^{-3}$ to $2.0 \times 10^{20} \mathrm{~m}^{-3}$, are accessible with the multi-pass scheme at the 2 nd harmonic of the ordinary mode (O2 scenario). It should be mentioned that the density cut-off for $\mathrm{O}$-mode propagation is $2.4 \times 10^{20} \mathrm{~m}^{-3}$, however for higher absorption lower densities are required. For heating of so-called over-dense plasma beyond the $\mathrm{O} 2$ cut-off density, the more sophisticated OXB scenario using electron Bernstein waves is foreseen. Additionally, a multipass X3 scenario at $140 \mathrm{GHz}$ and at $104 \mathrm{GHz}$ with reduced magnetic field is under consideration.

In the present work, the main heating scenarios for W7-X with the X2- and O2-modes are investigated by means of predictive simulations using a 1-D transport code [4] coupled self-consistently with the ray-tracing code TRAVIS [5]. A possible way for transition from $\mathrm{X} 2$ to $\mathrm{O} 2$ scenarios is also analyzed. Compensation of the bootstrap current by electron cyclotron current drive (ECCD) is also considered.

The present understanding and all theoretical assumptions adopted for modeling are based on the substantial experience from W7-AS and other experiments[6-8]. In calculations, neoclassical core confinement with empirical anomalous transport at the plasma edge is assumed. The anomalous diffusivity scales inversely with plasma density in the region of high density gradient and decays exponentially towards the plasma axis. The radial electric field, electron and ion temperatures are advanced

\footnotetext{
a e-mail: nikolai.marushchenko@ipp.mpg.de
}

This is an Open Access article distributed under the terms of the Creative Commons Attribution License 2.0, which permits unrestricted use, distribution, and reproduction in any medium, provided the original work is properly cited. 
self-consistently with a calculation of the power deposition profiles by the ray-tracing code TRAVIS. The shape of the density profile with a gradient region of about $10 \mathrm{~cm}$ is fixed. The modeling is performed for the "standard" magnetic configuration optimized for confinement.

\section{Low and moderate densities: X2 scenario}

The typical ECRH/ECCD scenario launching the X2-mode is most acceptable for low and moderate plasma densities [3]. Due to a high optical depth, $\tau_{\mathrm{X} 2} \gg 1$, the complete single-pass absorption is expected (if the mode-purity is sufficiently high). In optically thick plasmas, the location and width of the deposition profile for the RF-beam are defined predominantly by the magnetic configuration and are not very sensitive to the plasma profiles. Two physical effects are responsible for spatial location and width of the deposition profile, the Doppler shift for oblique launching and relativistic broadening.

In order to show the typical X2 scenario in W7-X, the following conditions were applied for simulations. Five RF-beams of $1 \mathrm{MW}$ each were launched obliquely from the different ports. The magnetic field on axis at the toroidal angle $\phi=0^{\circ}$ (here, the maximum of $B$ is located) was chosen $B_{0}=2.56 \mathrm{~T}$ (the "resonance" value for $140 \mathrm{GHz}$ is $2.5 \mathrm{~T}$ ) in order to have the deposition profile shifted in the low-field-side direction (this is desirable for compensation of finite $\beta$-effects and if the $\mathrm{X} 2$-scenario is applied as pre-heating scenario before switching to the $\mathrm{O} 2$-scenario).
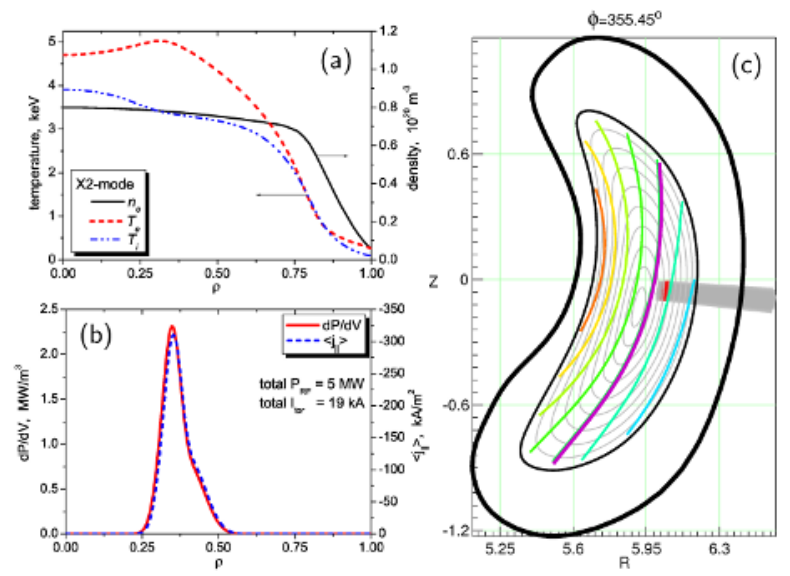

Fig. 1. Results for X2-scenario in W7-X: (a) plasma profiles calculated by coupled $1 \mathrm{D}$ transport code and ray-tracing code TRAVIS; (b) tottal deposition and current drive profiles from the five oblique beams launched from the different ports; (c) the ray trajectories in RZ-plane for one beam.

In the calculations, a density profile with $n_{e}(0)=0.8 \times 10^{20} \mathrm{~m}^{-3}$ (see Fig. 1 (a)) and uniform effective charge $Z_{\text {eff }}=1.5$ were fixed. The plasma temperatures (see Fig. 1 (a)) together with the radial electric field (not shown) were calculated under the assumption that neoclassical transport dominates in the plasma core while anomalous transport acts only in the periphery of plasma. In Fig. 1 (b), the deposition and current drive profiles obtained as the sum of all five beams are shown. As an example for one of the five beams, in Fig. 1 (c) the ray-trajectories are shown projected onto the $R Z$-plane in the maximum of deposition.

Since all beams are launched approximately at the same angles, the total deposition profile is well localized. One can also see that the shape of the current drive profile practically coincides with the deposition profile. This is natural for plasmas with high optical thickness, where the narrow deposition profile guarantees absorption by passing electrons alone and absence of the Ohkawa effect.

Summarizing the results for the X2 scenario for the start-up and preheating of plasmas with moderate density, one can conclude that this scenario is basically understood and no surprises are expected.

\section{High densities: 02 scenario}

Contrary to the X2-scenario, where single-pass absorption is complete and location of the deposition profile is defined by the magnetic field (location of the resonance) and obliqueness of the beam (Doppler shift), the O2-scenario is more complex. First of all, cyclotron absorption of the O2-mode is much weaker than of the X2-mode and for typical plasma parameters the plasma is optically gray $\left(\tau_{\mathrm{O} 2} \sim 1\right)$ with the single-pass absorption about $80-90 \%$. To improve an absorption, the multi-pass 
scenario is foreseen, where the second pass is proved by the mirrors installed at the inner wall [3]. Remaining power (usually about few percents) can be absorbed during the next passes after reflections from the wall.

During the reflection and re-entrance of the RF-beam into the plasma, a partial depolarization takes place, leading to reduction of the wave-mode purity in the next passes. In calculations, nevertheless, we assume the same polarization and take into account only three passes. This simplification is based on the assumption that the unpolarized fraction must be ultimately absorbed in the plasma after a number of passes. Even in the case of more precise knowledge about polarization of the reflected beam, not knowing the exact conditions for further reflections, no sense to calculate the number of passes more than three. Apart from this, an accurate calculation of deposition for this small rest fraction of power in optically gray plasmas is not necessary.

Since the absorption coefficient for the O2-mode is proportional to $T_{e}^{2}$, the O2-scenario cannot be applied as the start-up scenario and needs preheating. Considering the X2-scenario described in the previous subsection as a pre-heating scenario, after reaching the steady-state and changing the polarization from $\mathrm{X}$ - to $\mathrm{O}$-mode with the same launch conditions (beams must be directed to the mirrors), we discuss below the results of modeling of the $\mathrm{O} 2$-scenario with the density increased to $n_{e}(0)=1.2 \times 10^{20} \mathrm{~m}^{-3}$. Keeping in mind also that plasma pressure is not negligible, the magnetic configuration was calculated by VMEC for the same
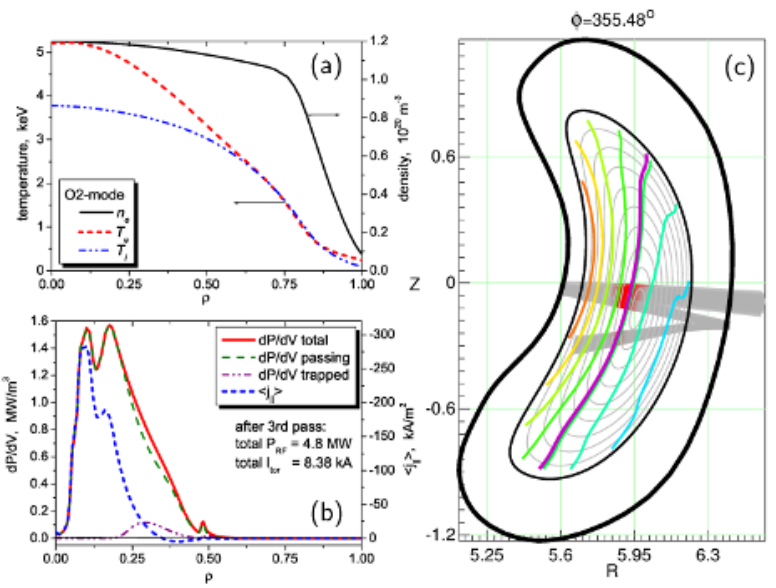

Fig. 2. The same as in Fig. 1 for the O2-scenario. vacuum field, but with $\langle\beta\rangle=2 \%$. The temperature profiles (see Fig. 2 (a)) are calculated in the similar manner as before, i.e. by a 1D transport code coupled with TRAVIS.

Contrary to the X2-scenario, in this case the deposition profile (see Fig. 2 (b)) is almost on-axis despite the same value of magnetic field. This is a consequence of two factors, finite $\beta$-effects (Shafranov shift and plasma diamagnetism) and the high sensitivity of the absorption on the electron temperature, which both significantly change the location and the shape of the deposition profile. As a result, the temperature profile is peaked on axis (compare with the flat profile in the case of X2-scenario, considered above) being well linked with the obtained deposition profile. Since single-pass absorption for the considered scenario is about $80 \%$, only $4 \%$ of the launched power is unabsorbed after three passes. An example of the ray-tracing results for the same beam as before is shown in Fig. 2 (c), where the trajectories are projected onto the $R Z$-plane in the maximum of deposition for the first pass.

The structure of the deposition and current drive profiles in the $\mathrm{O} 2$ scenario is much more complex than with the X2-scenario. In an optically gray plasma, where the cyclotron interaction is localized well neither in real nor momentum space, both the Ohkawa effect and contribution from electrons with $k_{\|} v_{\|}<0$ appear, which drive the current with opposite sign, reducing the total current. Apart from this, the trapped electrons which participate in absorption do not produce any current drive (here, possible contribution from the barely trapped electrons is neglected). As a result, the total toroidal current for the same launched power is more than a factor of two smaller in comparison with the $\mathrm{X} 2$-scenario.

\section{Transition from $\mathrm{X} 2$ to $\mathrm{O} 2$ scenarios}

In order to perform transition from $\mathrm{X} 2$ to $\mathrm{O} 2$ scenario, one needs to find first of all the conditions for stable operation with both scenarios. Two parameters have to be considered, magnetic field strength and density. Magnetic field is definitive for location of the deposition profile, while density is important for propagation and the rate of absorption. 

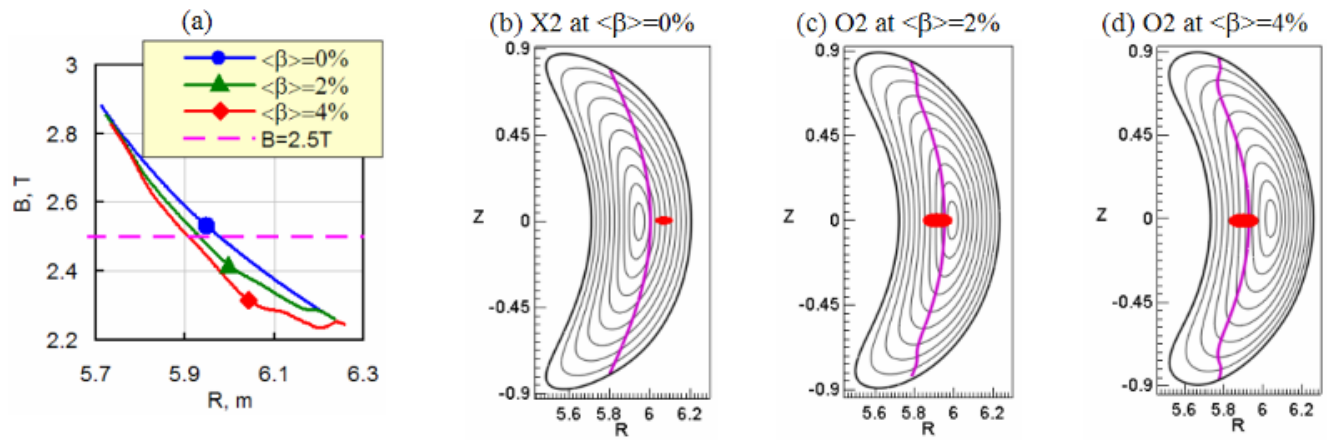

Fig. 3. (a): $B$-field along major radius, magnetic axis positions are shown by markers. (b-d): RZ cross-section in the "bean-shaped" plane with locations of the ECRH deposition for both X2 with vacuum magnetic configuration, $\langle\beta\rangle=0$ (b), and $\mathrm{O} 2$ scenarios with $\langle\beta\rangle=2 \%$ (c) and $4 \%$ (d).

For the X2 scenario, the upper density for operation must be chosen, $n_{e}<10^{20} \mathrm{~m}^{-3}$, that is somewhat below the cut-off density. This limitation excludes the case with strong refraction, which makes scenario too sensitive to the finite $\beta$-effects which can be critical for oblique propagation. For the $\mathrm{O} 2$ scenario, the lower density for operation can be found from the condition of sufficiently high (more than $80 \%$ ) single-pass absorption. For temperatures reached in the X2 scenario, this condition can be satisfied for $n_{e}>0.8 \times 10^{20} \mathrm{~m}^{-3}$, which overlaps well with the density range for X2.

Since finite $\beta$-effects change the magnetic configuration and shift the location of the deposition profile, the effect of finite $\beta$ needs to be carefully investigated. In Fig. 3 (a), the magnetic field-strength dependencies on volume averaged plasma $\langle\beta\rangle$ are shown. With increasing $\langle\beta\rangle$, the magnetic field at the axis is reduced from $2.56 \mathrm{~T}$ to $2.35 \mathrm{~T}$ due to the Shafranov shift and plasma diamagnetism. Fig. 3 (b-d) show schematically the power deposition zones which are depicted by mapping of deposition profiles to the corresponding flux surfaces. It is seen that the ECRH resonance region moves to the high-fieldside. In this case, $\mathrm{O} 2$ absorption decreases due to a shift of the resonance in the domain with smaller electron temperature leading to an additional decrease of the optical depth. For compensation of the finite $\beta$-effects, the magnetic field-strength, $B_{0}$, needs be chosen to optimize the absorption.

In order to find the optimal conditions for $\mathrm{X} 2 \rightarrow \mathrm{O} 2$ transition, a magnetic field scan is performed $\left(B_{0}\right.$-scan), checking all possible scenarios with on- and off-axis heating. In calculations, the density was kept the same, $n_{e}(0)=0.8 \times 10^{20} \mathrm{~m}^{-3}$, as well as the orientation of all five beams of $1 \mathrm{MW}$ each. The X2 scenario was calculated with both vacuum and $\langle\beta\rangle=2 \%$ magnetic configurations. The vacuum magnetic configuration was considered just to check the conditions for the start-up, since the predicted temperatures (apart from the case of heating only the periphery) are sufficiently high and the finite $\langle\beta\rangle$ effects becomes significant. For simulation of the transition, the steady-state for X 2 scenario was calculated for the given value of the magnetic field on axis (actually, the same magnetic equilibrium was applied with only the vacuum magnetic field $B_{0}$ rescaled). After this, the same was performed for the ordinary mode, i.e. for the $\mathrm{O} 2$ scenario, where three passes were taken into account. Additionally, the high density scenario, $n_{e}(0)=1.5 \times 10^{20} \mathrm{~m}^{-3}$, was calculated for full power $10 \mathrm{MW}$ and the magnetic equilibrium with $\langle\beta\rangle=4 \%$.

Analyzing the results, the range of $B_{0}$ values optimal for operation was found. As criteria for this optimum, heating within $\rho<0.5$ and sufficiently high temperatures in steady-state were chosen. Apart from this, the $\mathrm{O} 2$ scenario has an additional important factor for selection - the fraction of shinethrough radiation after three passes, and the acceptable range of magnetic field was defined from the condition $P_{\text {shine }} / P_{\text {inj }}<3 \%$. The results are presented in Fig. 4.

The optimal $B_{0}$ for X2 start-up and steady-state operation for both X2 and O2 with the same densities, power and equilibrium, are overlapping, but not identical. This scan confirms the preliminary conclusion that the best conditions for transition to $\mathrm{O} 2$ scenario with moderate density is the start-up and pre-heating with increased $B_{0}$, which shifts the resonance in the low-field-side direction. With this setup, the $\mathrm{O} 2$ experiments can be performed at the first phase of W7-X operation, where the inertially cooled divertor limits the pulse length $\Delta t<10 \mathrm{sec}$. 


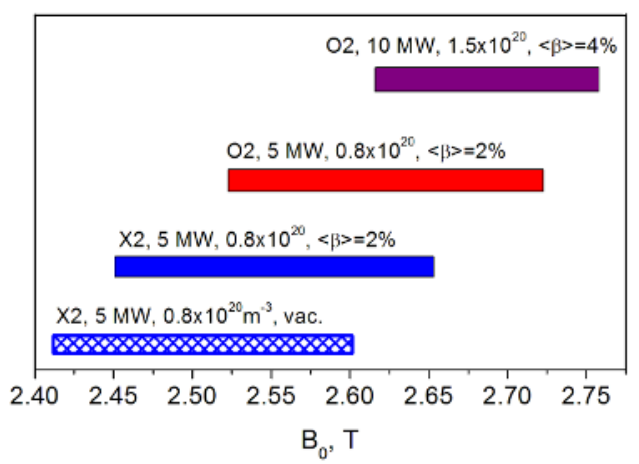

Fig. 4. Magnetic field range optimal for different scenarios with effective ECR heating and minimized the shine-through radiation.
Hovewer, the high-beta $\mathrm{O} 2$ scenario with full power has no overlap with the $B_{0}$-range defined for the startup. As a possible solution for the long-pulse operation $(\Delta t>10 \mathrm{~min})$, the optimized transition from X2 to $\mathrm{O} 2$ can be proposed. Since the current in superconducting coils can be slowly changed, the magnetic field in the device can be ramped up during the discharge with the rate up to $0.24 \mathrm{~T} / \mathrm{min}$ [9]. Then, the following scheme can be considered. After start-up and pre-heating with X2-mode (few seconds), the change of polarization from $\mathrm{X}$ - to O-mode has to be performed with simultaneous compensation of the finite $\beta$-effects by the growth of $B_{0}$ (one - two minutes). The density ramping-up and increasing of power also must be performed with the time-scale defined by the inertia of the superconducting coils. Since $\Delta B_{0} \simeq 0.2 \mathrm{~T}$ is quite sufficient for transition from the start-up conditions to the optimal conditions for steady-state operation in the high-density $\mathrm{O} 2$ scenario, this transition can be easily performed within a few minutes.

Summarizing the results for transition from X2- to O2-mode, one can conclude that the $\mathrm{O} 2$ scenario with moderate density can be easily performed in the first phase of W7-X operation, while the long operation with high-beta $\mathrm{O} 2$ scenario requires an additional investigations.

\section{Control of net plasma-current}

The plasma current, $I_{p}$, affects the edge value of the rotational transform, $\Delta t \simeq 1.2 \times 10^{-3} I_{p}[\mathrm{kA}]$, which may exceed the allowed range for a proper island divertor operation without external field compensation [10]. The acceptable shift of the X-point, $\Delta Z[\mathrm{~cm}] \simeq 0.3 I_{p}[\mathrm{kA}]$, is only a few centimeters, and the net plasma current is limited by $\left|I_{p}\right|<10 \mathrm{kA}[11]$. W7-X is not equipped with an Ohmic transformer, so the only source of external current for control needs is ECCD. Below, we present the results of predictive transport simulations for W7-X using the latest models for estimating bootstrap current [12] and ECCD [13] based on solution of the Spitzer problem with parallel momentum conservation taken into account.

A density scan has been performed for $5 \mathrm{MW}$ $\mathrm{X} 2$-mode $\left(n_{e}(0)<10^{20} \mathrm{~m}^{-3}\right)$ and $\mathrm{O} 2$-mode $\left(n_{e}(0)>10^{20} \mathrm{~m}^{-3}\right)$ for densities $(0.3-1.8) \times$ $10^{20} \mathrm{~m}^{-3}$ and the "standard" magnetic configuration calculated for $\langle\beta\rangle=2 \%$. Optimum launch angles are chosen with respect to ECCD efficiency. The typical power deposition profile for X2-mode heating has a width of about $0.1 \mathrm{~m}$ (see Fig. 1 (b)). The $\mathrm{O} 2$-mode heating profile is localized close to the magnetic axis within $r_{\mathrm{eff}}=0.20 \mathrm{~m}$ (see Fig. 2 (b)). As before, the shape of the density profile is held fixed and the whole profile is scaled during the scan. Again, the transport model is chosen to be a combination of neoclassical and simple anomalous models with the latter always dominant at the plasma edge.

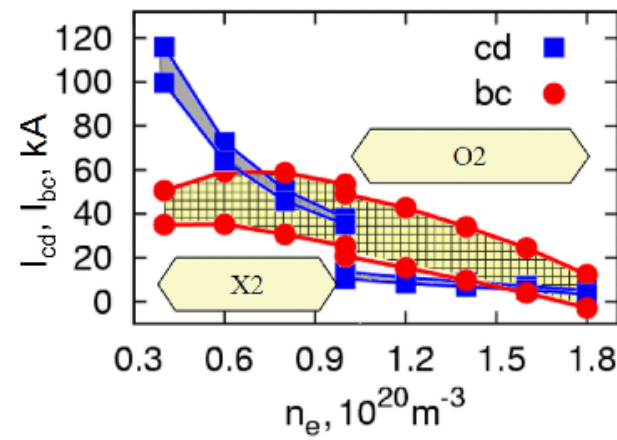

Fig. 5. Expected ranges of the bootstrap current (area with grid pattern) and the ECCD (grayed area) as a function of the plasma density for the X2-mode and $\mathrm{O} 2$-mode scenarios and $5 \mathrm{MW}$ ECR heating.

Two different levels of anomalous transport were simulated, one with neoclassical confinement dominant in the core, and the second with anomalous transport of the same order as neoclassical. As a consequence, the bootstrap current, $I_{\mathrm{bc}}$, presented in Fig. (5) is shown by two lines, indicating the area of possible values. Contrary to $I_{\mathrm{bc}}$, the current drive, $I_{\mathrm{cd}}$, is less sensitive to the plasma profiles 
(and transport models) being dependent mostly on the magnetic configuration. At the same time, the reduction of $I_{\mathrm{cd}}$ with changing of the scenario is much stronger being a consequence of quite different conditions in optically thick and gray plasmas.

One finds that for the X2 scenario with moderate density the bootstrap current can be easily compensated, while for the $\mathrm{O} 2$ scenario the efficiency of ECCD is not sufficient.

\section{Summary}

Different ECRH/ECCD scenarios have been analyzed using the self-consistently coupled ray-tracing and 1D transport codes. Only the "standard" magnetic configuration with improved neoclassical confinement was used here for modeling and all conclusions made below are for this configuration. For the X2 scenario, suitable for low and moderate densities, $n_{e}<1.0 \times 10^{20} \mathrm{~m}^{-3}$, good confinement with high temperatures is predicted. Similar results are found also for the $\mathrm{O} 2$ multi-pass scenario, for higher densities, $n_{e}<2.0 \times 10^{20} \mathrm{~m}^{-3}$. The best density range for transition from X2- to O2-mode is $0.8-1.0 \times 10^{20} \mathrm{~m}^{-3}$. For both scenarios the finite $\beta$-effects are very influential for the results.

In order to find the relevant conditions for $\mathrm{X} 2 \rightarrow \mathrm{O} 2$ transition, both $\mathrm{X} 2$ and $\mathrm{O} 2$ scenarios were simulated for the same initial parameters and for the different magnetic field values $\left(B_{0}\right.$-scan $)$. It was shown that the optimal $B_{0}$-ranges for X2 and $\mathrm{O} 2$ operation with the same density are overlapping, but do not coincide. In particular, transition to the $\mathrm{O} 2$ scenario at moderate density can be easily performed in the first phase of W7-X operation with the short pulse length, $\Delta t<10 \mathrm{sec}$. However, the long time operation $(\Delta t>10 \mathrm{~min})$ with high-beta $\mathrm{O} 2$ scenario and full power $(10 \mathrm{MW})$ requires an additional investigations since the $B_{0}$-range for this does not overlap with start-up range. As a possible solution, the optimized transition is proposed, by changing the magnetic field-strength together with the polarization over a time-scale at about one minute (the rate of ramping up the magnetic field is $\left.\left|d B_{0} / d t\right|<0.24 \mathrm{~T} / \mathrm{min}\right)$.

The reachable values of ECCD were estimated for both X2 and O2 scenarios. In high-density operation using the O2-mode, the bootstrap current exceeds the maximum ECCD. As a consequence, ECCD control of the bootstrap current in W7-X is possible only for the X2 scenario. With full current control by ECCD, only a few skin-times (i.e. about $10 \mathrm{sec}$ ) are necessary to obtain stationary conditions for optimum divertor operation. For the high-beta O2 scenarios, however, only the "high mirror" configuration allows for complete bootstrap current control by ECCD. Apart from this, another discharge scenario for the "standard" configuration can be chosen. In particular, the edge value of the rotational transform in the vacuum configuration can be reduced by the amount which is generated by difference of maximum ECCD and the bootstrap current in the final steady state. The evolution of the total plasma current scales on the $L / R$-time (i.e. several $10 \mathrm{sec}$ ). Such a discharge scenario, however, is much more complex compared to the case of full ECCD control of the bootstrap current requires further investigation.

\section{References}

1. C. D. Beidler, et al., Fusion Technol. 17, (1990) 148

2. H. Maaßberg, W. Lotz, and J. Nührenberg, Phys. Fluids B 5, (1993) 3728

3. V. Erckmann, et al., Fusion Sci. Technol. 52, (2007) 291

4. Yu. Turkin, et al., Fusion Technol. 50, (2006) 387

5. N. B. Marushchenko, et al., Jpn. J. Plasma Fusion Res. 2, (2007) S1000

6. V. Erckmann and U. Gasparino, Plasma Phys. Control. Fusion 36, (1994) 1869

7. V. Erckmann, et al., Fusion Eng. Design 53, (2001) 265

8. H. Maaßberg, et al., Plasma Phys. Control. Fusion 37, (2005) 1137

9. Th. Rummel, Th. Mönnich, M. Köppen, and E. Harmeyer, Fusion Eng. Design 84, (2009) 1658

10. R. Koenig et al., Plasma Phys. Control. Fusion 44, (2002) 2365

11. A. Werner et al., Rev. Sci. Instrum. 79, (2008) 10F122

12. H. Maaßberg, C.D. Beidler and Y. Turkin, Phys. Plasmas 16, (2009) 072504

13. N. B. Marushchenko, et al., Fusion Sci. Technol. 55, (2009) 180 